\title{
Logical Analysis of Formative Measurement
}

\author{
George R. Franke
}

\begin{abstract}
This paper applies deductive logic to two fundamental premises of formative measurement: that formative constructs are linear combinations of their indicators, and that they have linear effects on their outcomes. These premises lead to several important conclusions: (1) Formative constructs are determined as much by their outcomes as by their indicators; (2) formative constructs completely mediate the effects of their indicators on their outcomes; (3) using reflective indicators as outcomes in formative models does not test the formative conceptualization; (4) the formative disturbance term plays no meaningful role in estimating or interpreting formative models; (5) formative models need not account for the correlations between the outcome variables, and (6) allowing correlated measurement errors between the outcomes make formative measurement models equivalent to canonical correlation analyses such that (7) the causal influences and roles of indicators versus outcomes are ambiguous. These results logically call for changes in how researchers conceptualize, analyze, and interpret formative measurement models.
\end{abstract}

\section{References are available upon request.}

G.R. Franke $(\bowtie)$

University of Alabama, Tuscaloosa, AL, USA

e-mail: gfranke@cba.ua.edu 